\title{
INCIDENCE AND ANTIMICROBIAL SUSCEPTIBILITY PATTERN OF NEONATAL SEPTICAEMIA FROM TERTIARY CARE HOSPITAL OF INDIA
}

\author{
DHUMAL P., UJAGARE M., GANDHAM N., NAGDAWANE R.P., SARDAR M., SHARMA M. AND JADHAV S.V.* \\ Department of Microbiology, Pad. Dr. D.Y. Patil Medical College and Hospital, Pimpri, Pune-18, MS, India. \\ *Corresponding Author: Email- patilsv78@gmail.com
}

Received: September 20, 2012; Accepted: September 27, 2012

\begin{abstract}
-
Introduction- Septicemia is a clinical syndrome characterized by fever, malaise, tachycardia, hyperventilation and toxicity or prostration, which results when circulating bacteria multiply at a rate that exceeds removal by phagocytes. "Failure to thrive" may indicate chronic septicemia in infants. Neonatal sepsis is an important cause of neonatal morbidity and mortality. In India nearly one fourth of half of neonatal deaths is due to neonatal septicemia.

Materials and Methods- The study was approved by the Institute Ethical committee and conducted between January 2006 to December 2007. 50 infants with clinically suspected neonatal septicaemia were included in the present study. An incidence of clinically suspected septicaemia with positive blood cultures, their bacteriological profile and sensitivity pattern were studied.

Results and observation- Incidence of culturally positive neonatal septicaemia in NICU is $62 \%$ out of clinically suspected neonates. Male neonates may be prone to septicaemia. There is a correlation between preterm babies and neonatal septicaemia. CRP, band cell \% and band cell to neutrophil ratio are significant parameters for early diagnosis. Local bacterial infections are less significant with bacteremia. Gram negative bacteria are showing increase in resistance towards antibiotics. In vitro testing, most bacteria have good sensitivity towards ciprofloxacin and Amikacin.

Conclusion-Study reveals elevated incidence of neonatal septicaemia in our hospital. To reduce comprehensive neonatal mortality, strategies of proven value such as hand hygiene, barrier nursing, and constraint use of antibiotic have to be implanted.
\end{abstract}

Keywords- Neonatal septicaemia, Blood culture, Klebseilla spp., E. coli

Citation: Dhumal P., et al. (2012) Incidence and Antimicrobial Susceptibility Pattern of Neonatal Septicaemia from Tertiary Care Hospital of India. International Journal of Medical and Clinical Research, ISSN: 0976-5530 \& E-ISSN: 0976-5549, Volume 3, Issue 7, pp.-207-211.

Copyright: Copyright@2012 Dhumal P., et al. This is an open-access article distributed under the terms of the Creative Commons Attribution License, which permits unrestricted use, distribution and reproduction in any medium, provided the original author and source are credited.

\section{Introduction}

Septicemia is a clinical syndrome characterized by fever, malaise, tachycardia, hyperventilation and toxicity or prostration, which results when circulating bacteria multiply at a rate that exceeds removal by phagocytes. "Failure to thrive" may indicate chronic septicemia in infants $[1,2]$. Neonatal sepsis is an important cause of neonatal morbidity and mortality [3,4]. In India nearly one fourth of neonatal deaths is due to neonatal septicemia [5]. It is defined as a clinical syndrome characterized by signs of systemic infections and documented bacteremia in the first month of life [1-5]. The normal fetus is sterile until shortly before birth as the placenta and amniotic sac are highly effective barriers to infections. At birth, the newborn loses the protection afforded to it in the uterus and gets exposed to the microbial world [6]. Ascending infection due to disruption of amniotic membranes, infection upon passing through birth canal, exposure to infected blood at delivery and transplacental transmis- sion of infection are some of the major causes of neonatal septicemia [7]. Neonatal conditions such as preterm, low birth weight (LBW), male sex and twin gestation appear to be risk factors too [8]. The incidence and etiology of the organism causing septicemia varies from nursery to nursery and depends on factors such as handling of babies, use of contaminated equipments, sinks, incubators, etc. Prevention and cure of neonatal septicemia is possible by early diagnosis followed by proper antibiotic therapy and avoidance of cross infection in nurseries [6-9]. There are a number of indirect methods to detect bacteremia such as buffy coat, total and differential leucocyte count. But the definite diagnosis is isolation of microbes from blood culture [6-9]. Blood culture is a simple, effective and inexpensive means for identification of bacteria in blood and it is the diagnostic tool for bacteremia and septicemia [9-11]. A delay in antibiotic therapy can lead to several complications. Therefore an early diagnosis and judicious use of antibiotics for treatment is essential. The present study was undertaken to find out the 
current incidence, bacteriological profile and antibiotic sensitivity pattern of neonatal septicemia in Pad. Dr. D.Y. Patil Medical College \& Hospital, Pimpri, Pune-18.

\section{Aims and Objectives}

The present study was undertaken with the following aims- To find the incidence of positive blood cultures in clinically suspected cases of septicemia. To characterize and identify isolates obtained by culturing blood specimens in clinically diagnosed cases of septicemia. To find antibiotic susceptibility pattern of isolates.

\section{Materials and Methods}

The study was approved by the Institute Ethical committee and conducted between January 2006 to December 2007. The clinical specimen were obtained from neonatal intensive care unit (NICU). Clinico-demographic data of study patients was noted. The specimens were processed according to standard conventional bacteriological methods and bacterial pathogens were identified and confirmed by standard biochemical tests [12-17].

\section{Results and Observation}

50 infants with clinically suspected neonatal septicaemia were included in the present study [Table-1]

Table 1- Incidence of Neonatal Septicemia.

\begin{tabular}{|cc|}
\hline Total no. Of Clinically Septicemia Infants Culturally positive infants $(n \%)$ \\
50 & $31(62 \%)$ \\
\hline
\end{tabular}

In the study conducted, out of 50 clinically suspected septicemic patients, $31(62 \%)$ had bacteriologically positive blood cultures [Table-2].

Table 2- Relation of term with Septicemia

\begin{tabular}{|lcc|}
\hline Term $\%$ & Clinically septicemic infants & Culturally positive infants N=31 \\
\hline Pre term & $26(52 \%)$ & $18(58.06 \%)$ \\
Full term & $24(48 \%)$ & $13(41.93 \%)$ \\
\hline
\end{tabular}

In clinically suspected septicaemic infants, 26(52\%) and 24(48\%) were preterm and full term respectively. While in culturally positive neonates, $18(58.06 \%)$ and $13(41.93 \%)$ were preterm and full term respectively. There is a slight predominance of septicaemia in preterm babies [Table-3].

Table 3-Relation of sex with Neonatal Septicemia

\begin{tabular}{|lcc|}
\hline Term $\%$ & Clinically septicemic infants & Culturally positive infants N=31 \\
Pre term & $29(58 \%)$ & $22(70.09 \%)$ \\
Full term & $21(42 \%)$ & $9(29.03 \%)$ \\
\hline
\end{tabular}

Male predominance is observed in both clinically suspected (58\%) as well as culturally positive (70.9\%) infants [Table-4].

Table 4- Various indirect methods in septicaemic group of neonates

\begin{tabular}{|lccc|} 
Parameters & & Proved N=31 & Probable N=19 \\
\hline CRP > 6mg/dl & $+\mathrm{ve}$ & $19(61.2 \%)$ & $6(31.57 \%)$ \\
& $-\mathrm{ve}$ & $12(38.7 \%)$ & $13(68.42 \%)$ \\
TLC & $<11000$ & $18(58.06 \%)$ & $8(42.1 \%)$ \\
& $11000-18000$ & $11(35.48 \%)$ & $11(57.8 \%)$ \\
\multirow{2}{*}{ Band cells (\%) } & $>18000$ & $2(6.45 \%)$ & 0 \\
& $0-10$ & $8(25.8 \%)$ & $14(73.6 \%)$ \\
Band cells/Neutrophil ratio & Oct-20 & $10(32.25 \%)$ & $5(26.3 \%)$ \\
& $>20$ & $13(41.9 \%)$ & 0 \\
& $<0.2$ & $11(35.48 \%)$ & $14(73.6 \%)$ \\
& $>=0.2$ & $20(64.51 \%)$ & $5(26.3 \%)$ \\
\hline
\end{tabular}

Blood culture was done for all 50 cases, out of which 31 were culturally positive and was taken as "proved" septicaemic, while the remaining 19 were taken as "probable" septicaemic. CRP concentration $>6 \mathrm{mg} / \mathrm{dl}$ is taken as positive test and detected $61.2 \%$ and $31.5 \%$ in proved and probable septicaemia respectively. Leucopenia $(<11000)$ was found in $58.06 \%$ and $42.10 \%$ in proved and probable septicaemia respectively. Leucocytosis was found in $6.45 \%$ of "proved" cases as well. Band cells $>10 \%$ observed in proved and probable septicaemic infants as $23(74.19 \%)$ and $5(26.3 \%)$ respectively. In the present study band cell to neutrophil ratio $(>0.2)$ was found in $64.51 \%$ of proved and $26.3 \%$ of probable cases [Table-5].

Table 5- Evaluation of each individual indirect test

\begin{tabular}{|lcccc|}
\hline Feature & Sensitivity & Specificity & PPA & NPA \\
\hline CRP $>$ 6mg/dl & $61.20 \%$ & $68.42 \%$ & $76 \%$ & $86.60 \%$ \\
Leucopenia & $58.06 \%$ & $57.89 \%$ & $69.20 \%$ & $50 \%$ \\
Band cells (\%) & $74.19 \%$ & $73.68 \%$ & $82.14 \%$ & $63.60 \%$ \\
Band cells/Neutrophil ratio (>=0.2) & $80 \%$ & $73.68 \%$ & $86.90 \%$ & $56 \%$ \\
\hline
\end{tabular}

PPA- Positive Predictive Accuracy

NPA- Negative Predictive Accuracy

\section{Discussion}

50 infants with clinically suspected neonatal septicaemia were included in the present study. An incidence of clinically suspected septicaemia with positive blood cultures, their bacteriological profile and sensitivity pattern were studied. The efficacy of the septicaemia screen with different indirect methods was also included in the study.

\section{Incidence}

Out of 50 clinically suspected cases of neonatal septicaemia, 31 $(62 \%)$ infants were confirmed by positive blood cultures while 9 (18\%) showed positive cultures from localized infections [Table-1]. Roy, et al reported $47.5 \%$ positive blood cultures. Monga, et al and Sharma, et al reported the incidence of clinical septicaemia in their units as $26.3 \%$ and $33.7 \%$ respectively. Guha, et al observed clinical septicaemia in the 24.35 infants and $2.8 \%$ showed positive blood cultures. Various workers have reported incidence in which positive blood cultures vary from 15 to $75 \%$ in clinically septicaemic infants respectively [18-21].

\section{Term}

Clinically neonates suspected to have septicaemia, $52 \%$ were preterm delivery out of that $58.06 \%$ were positive blood culture. Predominance of preterm babies in case of cultural positivity was evident [Table-2]. Bhakoo, et al reported only 15\% neonates were preterm while Saxena, et al and Choudhary, et al reported prematurity in $29.4 \%$ and $46 \%$ respectively. Other workers have stated high incidence on septicemia in preterm. Anand, et al reported predominance of preterm infants $62.1 \%$ in septicemia with coagulase negative Staphylococcus. William, et al reported mortality in very low birth weight infants with early onset systemic infections is close to $40 \%$, three times higher than in infants of the same gestation without infection. There are many causes for higher incidence of preterm in septicaemia. Complications of labor and resuscitation thereafter are common in preterm and L.B.W babies. These neonates require more intensive care management after birth. Newly born neonates are unable to concentrate the inflammatory cells selectively at the site of infection. These observations were found 
more commonly in premature babies [22-27].

\section{Gender}

Out of 50 clinically suspected cases of neonatal septicaemia, 29 $(58 \%)$ were males and $21(42 \%)$ were females. Out of 31 culturally positive cases, $22(70.9 \%)$ were males where as only $9(29.03 \%)$ were females. Here a clear male predominance is evident [Table3]. Other studies have also reported a high incidence of septicemia in male neonates $[14,35]$. Saxena, et al reported male predominance in $59.17 \%$ cases [14]. Gupta, et al reported male predominance in $64.7 \%$ neonatal septicemia caused by Klebsiella [9]. An increased incidence of septicaemia in male infants than female infants may be due to the presence of immunoregulatory genes located on "X" chromosome [34] or probably because of more attention paid by the parents to male neonates in our country [28-31].

\section{Evaluation of Sepsis Screen in the Early Diagnosis of Neonatal Septicaemia}

In a study group of 50 clinically suspected septicemic infants, blood cultures were positive in 31 neonates and these taken as "proved" or definite septicaemic. Remaining 19 were taken as "probable" septicaemic [Table-4]. The frequency distribution of various indirect methods studied were as follows:

- C-reactive protein (CRP)- was detected positive in 19(61.2\%) and6 (31.57\%) in proved and probable septicaemic cases.

- Total leucocyte count of $<11000$ cubic $\mathrm{mm}$ were detected in $58.06 \%$ and $42.1 \%$ in proved and probable septicaemic cases respectively.

- Occurence of band cells $>10 \%$ were $74.15 \%$ in proved and $26.3 \%$ in probable septicaemic cases.

- Band cells to neutrophil ratio $(\mathrm{BC} / \mathrm{N})>0.2$ was obtained in $64.15 \%$ and 26.3 in proved and probable cases respectively.

Evaluation of single test in terms of sensitivity, specificity, positive predictive accuracy and negative predictive accuracy were given in [Table-5]. In the present study CRP, Band cells and Band cell to neutrophil ratio were found to be most sensitive parameters in septicemia. Their sensitivity being $61.2 \%, 64.5$ and $74.19 \%$ respectively. Leucopenia had a sensitivity of $58.06 \%$. The positive predictive accuracy for septicaemia was detected more in $\mathrm{BC} / \mathrm{N}$ ratio (86.9\%) while minimum was located in leucopenia (69.2\%). The usefulness of the various tests in early detection of neonatal septicaemia is controversial. As a single test they have less predictive accuracy but if combined together they may be useful in detecting septicaemia. Mishra, et al found $\mathrm{BC} / \mathrm{N}$ ratio had $90 \%$ sensitivity and of that of CRP had only $22 \%$. R.S. Jaiswal, et al showed that the correlation between positive CRP positive blood culture was significant $(p<0.005)$. Sharma, et al and Singh, et al reported $80 \%$ sensitivity and approximately $90 \%$ specificity for CRP estimation alone. Russel, et al concluded that CRP is an early indicator of infection in neonates, Bhandari, et al observed that septicaemic screen was more effective when performed after 1 st day of life. If done at birth it was found to be only $16.6 \%$ sensitive. Feigin, et al did not give significance to all above mentioned tests. They found counter immunoelectrophoresis (CIE) and limulus lysate assay having $90 \%$ predictive accuracy in diagnosing septicaemia [30-32].

\section{Bacteriological Profile of Neonatal Septicaemia}

In $61 \%$ cases, culture showed gram negative organisms. The predominant organism isolated in the present study was Klebseilla spp. (42.5\%) Staphylococcus aureus (32.5\%) and E. coli (12.5\%) respectively [Table-6]. Various workers reported the isolation of gram negative organisms from blood in 57 to $92.5 \%$ cases. Bhakoo, et al reported predominance of gram negative organisms in $57 \%$ cases while Vaidya reported it as $92.5 \%$. Sharma, et al observed gram negative bacilli constituted $81 \%$ of the culture growth. Vesicari, et al and Freedman found gram positive organisms as the predominant agent. Singh, et al reported the isolation of Klebseilla spp. in $46.7 \%$ followed with Staphylococcus albus in $11.9 \%$ and Enterobacter in $11.9 \%$ cases. Choudhury, et al isolated Klebseilla spp. in $27.8 \%$, coagulase positive Staphylococcus in $22.9 \%$ and $E$ coli in $22.9 \%$ Guha, et al found $40 \%$ E. coli, $26.2 \%$ Klebseilla spp. and $22.5 \%$ Staphylococcus. Out of 12 pus samples, 8 were positive by culture. The major isolates being Staphylococcus aureus and Klebseilla pneumoniae, 50\% each. In 6 cases $(75 \%)$ the same organism was isolated from blood, 4 Staphylococcus spp. and 2 Klebseilla spp. Total 3 CSF samples were studied each one was negative for culture. But blood culture of only one infant was positive for Klebsiella spp and rectal swab of another baby was positive. Out of 2 samples of rectal swab, one was positive for Klebseilla spp. but blood culture of the same infant was negative for growth. Namdeo, et al observed Klebsiella spp. was the commonest organism grown from CSF cultures. He also observed that infections were mostly caused by gram positive cocci (55\%), E. coli (27\%) and Pseudomonas spp. Ghanshyam, et al isolated $33.8 \%$ Klebsiella spp. followed by $24.4 \%$ Staphylococcus aureus. K Ako-Nai, et al reported that Staphylococcus aureus $33.8 \%$ as the predominant bacteria cultured from proven cases of septicaemia [29-32].

Table 6- Organism isolated from different sources

\begin{tabular}{|c|c|c|c|c|c|}
\hline $\begin{array}{l}\text { Organism } \\
\text { isolated }\end{array}$ & Blood N=50 & Pus $\mathrm{N}=12$ & $\begin{array}{c}\text { Stool/Rectal } \\
\text { swab (2) }\end{array}$ & CSF -3 & Total $N=67$ \\
\hline S. aureus & $9(29 \%)$ & $4(50 \%)$ & - & - & 13 \\
\hline CONS & $2(6.44 \%)$ & - & - & - & 2 \\
\hline Klebsiella spp & $12(35.48 \%)$ & $4(50 \%)$ & 1 & - & 17 \\
\hline E. coli & $5(16.12 \%)$ & 0 & 0 & 0 & 5 \\
\hline Acinetobacter & $1(3.22 \%)$ & - & - & - & 1 \\
\hline \multirow{3}{*}{$\begin{array}{l}\text { Pseudomonas } \\
\text { S. agalactae } \\
\text { (group B) }\end{array}$} & $1(3.22 \%)$ & - & - & - & 1 \\
\hline & $1(3.22 \%)$ & - & - & - & 1 \\
\hline & $31(62 \%)$ & $8(66.6 \%)$ & $1(50 \%)$ & 0 & $39(58.2 \%)$ \\
\hline
\end{tabular}

\section{Antibiotic Sensitivity Pattern}

The organism isolated from different cultured samples of 31 babies with their sensitivity pattern towards different antibiotics is enlisted in [Table-7]. All isolates were tested for 11 different antibiotics. Almost all gram negative bacteria were observed to be resistant towards first generation antibiotics. Gram positive organisms was sensitive towards all antibiotics. Streptococcus spp. was found to be sensitive to most antibiotics (90\%), while staphylococcus had a sensitivity of 70\%. Pseudomonas spp., Coagulase negative Staphylococcus and Acinetobacter spp. were most resistant, 50, 50\% and $27 \%$ respectively Streptococcus spp., Klebseilla spp., E coli and Acinetobacter spp. were found to be highly sensitivity to Ciprofloxacin (100\%, 78.5\%, 80\% and 100\% respectively). Staphylococcus aureus showed $92.3 \%$ sensitivity towards Cloxacillin, 
Table 7- Antimicrobial susceptibility of isolated strains.

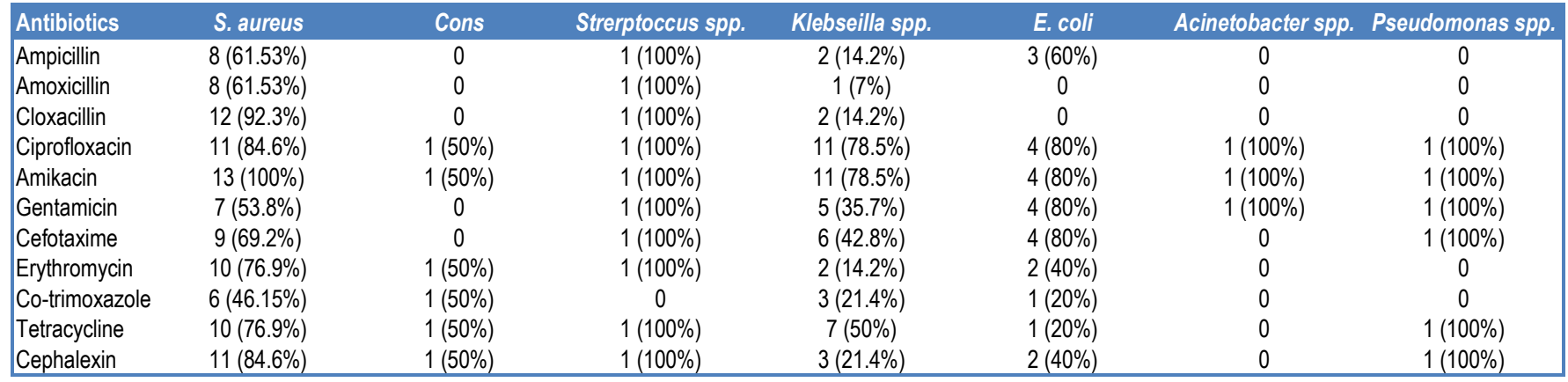

where as Coagulase negative Staphylococcus aureus was resistant to it. $E$ coli showed sensitivity towards ciprofloxacin, amikacin, gentamicin and cefotaxime while it showed resistance towards amoxicillin and Cloxacillin. Monga, et al reported a steady rise in resistant organisms, approximately $15 \%$ in 1981 to $35 \%$ in 1984 . Approximately $60 \%$ of the gram negative and $35 \%$ of gram positive organisms are reported resistant to penicillin, erythromycin, Gentamicin by Sharma, et al. Mathur, et al reported 3 to $5 \%$ and 40 to $60 \%$ of gram negative and 23 to $70 \%$ of gram positive organisms were sensitive towards Ampicillin and Gentamycin respectively. Anand, et al observed 1.34\% Coagulase negative Staphylococcus aureus were resistant to all antibiotics and sensitivity was maximum with newer cephalosporins, ciprofloxacin and Amikacin, 50\% each [33-37].

\section{Conclusion}

The fulminate nature of neonatal septicaemia and its high mortality rate has always posed a challenge to the skills of Microbiologists and pediatricians. The outcome of neonates with septicaemia largely depends on its early diagnosis. The clinical diagnosis remains a difficult task and is mimicked by various non-specific conditions. Emerging new resistant bacteria are also adding a new dimension to these problems. Study reveals elevated incidence of neonatal septicaemia in our hospital. To reduce comprehensive neonatal mortality, strategies of proven value such as hand hygiene, barrier nursing, and constraint use of antibiotic have to be implanted

\section{References}

[1] Moreno M.T., Vargas S., Poveda R. (1994) Padia. Infect. Dis. J., 13, 516-520.

[2] Weber M.W., Carlin J.B., Gatachalian S. (2003) Padia. Infect. Dis. J., 22, 711-716.

[3] Mc Cracken G.H. and Siegel J.D. (1981) New Engl. J. Med., 304, 642-647.

[4] Chaudhari P., Shrivastava D., Agarwal L. (1975) Indian J. Pedia.,12, 459-464.

[5] Guha D.K., Jaspal D., Das K. (1978) Indian J. Pedia., 15, 423428.

[6] Gupta P., Murali M.V., Kaul P.B., et al. (1993) Indian J. Pedia., 60, 565-572.

[7] Vaidya U., Bhave B., Hegde D., et al. (2004) Indian J. Pedia., 50, 103-104.
[8] Bakoo O.N. and Agarwal K.C. (1974) Indian J. Pedia., 111, 519 $-528$.

[9] Mishra J.N., Rai M.G., Chakraborty S., et al. (1985) Indian J. Pedia., 22, 281-286.

[10]Maxwell F.C. and Bourchier D. (1991) New Engl. J. Med., 23 104, 446-448.

[11]Saxena S., Anand N.K., Saini L., et al. (1980) Indian J. Pedia., $17,1-17$

[12]Malhotra N., Kumar A., Chansoria M. (1985) Indian J. Pedia., 22, 275-281.

[13]Singh M. (1978) Indian J. Pedia., 45, 314-318.

[14]Naeye R.L. (1979) New Engl. J. Med., 300,819-823.

[15]Boyes K.M., Gotoff S.P. (1986) New Engl. J. Med., 314, 16651669.

[16]Ralph A., Fransciosi (1972) Am. J. Clin. Pathol., 57, 215-219.

[17]Bannon M.J., Switchfield R.P., Weinding A.M. (1986) Arch. Dis. Child., 60, 360.

[18]Kishor K., Deorari A.K., Singh M. (1987) Indian J. Pedia., 24, 45-48.

[19]Smith J.A., Jennison R.P., Langley P.A. (1956) Lancet, 2, 903909

[20]Gluck L., Wood H.F., Fousek M.D., et al. (1966) Pedia. Clin. North Am., 13,1131

[21]Stoerner J.W., Pickering L.K., et al. (1978) Indian J. Pedia., 93, 864-74

[22]Schlegal R.J., Bellani J.A. (1969) Lancet, 2, 826-830

[23]Edward M.S., Jackson C.V., Baker C.J. (1981) JAMA, 254, 204 -210 .

[24]Diagnosis of Neonatal Sepsis (2004) Clin. Chem. 50(2).

[25]Ghyanshyam D., Kumhar, et al. (2002) J. Health Popul. Nutr. 20, 343-347.

[26]Roy I., Jain A., Kumar M., et al. (2002) Indian J. Med. Micrbiol., 3, 156-159.

[27]Vergnanol S., Sharland M., et al. (2005) Arch. Dis. Child Fetal Neonatal Ed., 90, 220-224

[28]Jaiswal R.S., Kaushal R.K., Goel A., et al. (2003) Indian J. Pedia., 40, 880-883.

[29]Akonai K., Adejuyigbe J., Ajayi V., et al. (1999) J. Trop. Pedia., 
45, 146-151.

[30]McGuire W., Clerihew L., Fowlie P.W. (2004) Br. Med. J., 329, 1277-1280.

[31]Issacs D., Barfield C., Clothier T., et al. (1996) J. Paedia. Child Health, 32, 158-161

[32]Barabar J., Stoll M.D., Hansen N., et al. (2002) Paediatrics, 110, 285-291.

[33]Livermore D.M. (1995) Clin. Microbiol. Rev., 8, 557-84.

[34]Desimoni M.C., Esquivel G.P., Merino L.A. (2004) Enferm Infec. Microbiol. Clin., 22, 507-511.

[35]Elemam A., Rahimian J., Mandell W., (2009) Clin. Infect. Dis., 49(2), 271-274.

[36]Bush K., Jacoby G.A., Medeiros A.A. (1995) Antimicrob. Agents Chemother., 39, 1211-33.

[37]Jain A., Roy I., Gupta M.K., Kumar M., Agarwal S.K. (2003) J. Med. Microbiol., 52, 421-5.

[38]Shukla I. (2004) Indian J. Med. Microbiol., 22, 87-91. 